Chin. J. Astron. Astrophys. Vol.0 (200x) No.0, 000-000

(http://www.chjaa.org )

Chinese Journal of

Astronomy and

Astrophysics

\title{
Monopole-charged pulsars and relevant issues
}

\author{
Xiao-Hong Cui, You-Ling Yue, Ren-Xin Xu, Guo-Jun Qiao \\ Astronomy Department, School of Physics, Peking University, Beijing 100871, China \\ Received ; accepted
}

\begin{abstract}
The aligned pulsars whose rotation axes and magnetic dipole axes are parallel should be positively charged. The total charge of pulsars is calculated after considering the electromagnetic field in and out the star under a specific condition. The statistical relation between the pulsar's rotation energy loss rate (or the period derivative) and the period may hint that the millisecond radio pulsars with small periods could be low-mass bare strange stars.
\end{abstract}

Key words: pulsars: general — stars: neutron — dense matter

\section{INTRODUCTION}

The study of pulsar magnetosphere is essential for us to understand various radiative processes, and thus observed emission in different bands. The charge-separated plasma (Goldreich and Julian 1969) in the magnetosphere of various gap models (Ruderman \& Sutherland 1975; Arons \& Scharlemenn 1979; Cheng, Ho, \& Ruderman 1986; Qiao et al. 2004) has been investigated.

After investigating the electromagnetic field of an aligned pulsar whose rotation axis is parallel to the magnetic axis, Xu, Cui \& Qiao (2006) suggested that these pulsars should be positively monopole-charged. We would like to study further charged pulsars in this paper. Supposing the pulsar is magnetized homogenously, we calculate the total charges $Q$. Provided that this charge is proportional to the radiation of pulsars, $Q$ should be proportional to $\dot{E}$, the spin down energy loss rate. The statistical relation between the pulsar's $\dot{E}$ (or the period derivative $\dot{P}$ ) and the period may hint that the millisecond radio pulsars with small periods could be low-mass bare strange stars.

This paper is arranged as follows. The total charge of pulsars is estimated in $\S 2$. In $\S 3$, we show possible evidence for low-mass millisecond pulsars with small $P$ by observational data. Conclusions are presented in $\S 4$.

\section{PULSAR'S CHARGE ESTIMATED}

If the pulsar is assumed to be magnetized homogenously, its magnetic field could be described as

$$
\boldsymbol{B}_{\text {in }}=\frac{8 \pi}{3} \boldsymbol{M}=\frac{2 \boldsymbol{m}}{r_{0}^{3}} \quad\left(r<r_{0}\right)
$$

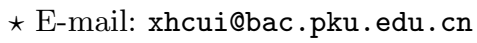


and

$$
\boldsymbol{B}_{\text {out }}=\frac{3 \hat{r}(\hat{r} \cdot \boldsymbol{m})-\boldsymbol{m}}{r^{3}} \quad\left(r>r_{0}\right),
$$

where $\boldsymbol{M}$ is the magnetized intensity, $\boldsymbol{m}$ is the magnetic dipole moment, $r_{0}$ is the radius of pulsar, $r$ is the distance from one arbitrary point in space to the stellar core, $\hat{r}$ is the corresponding unit vector. Here we define $\left|\boldsymbol{B}_{\text {in }}\right| \equiv B_{0}$.

If all the particles in and out the pulsar rotate synchronously around the magnetic axis, the electric field, $\boldsymbol{E}$, could satisfy

$$
\boldsymbol{E}+\frac{\boldsymbol{\Omega} \times \boldsymbol{r}}{c} \times \boldsymbol{B}=0
$$

without considering the magnetic field induced by synchronously rotating particles, where $\boldsymbol{\Omega}$ is the angular velocity of star rotating around the dipole rotation axis, which relates with rotating period of star $P$ by $P=2 \pi / \Omega$. If $\hat{k}$ is the unit vector of angular momentum, $\boldsymbol{\Omega}=\Omega \hat{k}$ and $\boldsymbol{m}=m \hat{k}$ for aligned pulsars. We estimate the charge under this specific condition.

From Eqs.(11) to (3), the electric fields in and out a pulsar are, respectively,

$$
\begin{gathered}
\boldsymbol{E}_{\text {in }}=\frac{\Omega r B_{0}}{c} \hat{k} \times(\hat{k} \times \hat{r}) \quad\left(r<r_{0}\right), \\
\boldsymbol{E}_{\text {out }}=\frac{\Omega r_{0}^{3} B_{0}}{2 c r^{2}}[3(\hat{k} \cdot \hat{r}) \hat{r}-\hat{k}] \times(\hat{k} \times \hat{r}) \quad\left(r>r_{0}\right) .
\end{gathered}
$$

The corresponding charges then could be

$$
Q_{\text {in }}=\rho_{\text {in }} \frac{4 \pi r_{0}^{3}}{3}=\frac{\nabla \cdot \boldsymbol{E}_{\text {in }}}{4 \pi} \frac{4 \pi r_{0}^{3}}{3}=-\frac{2 r_{0}^{3} \Omega B_{0}}{3 c},
$$

and $Q_{\text {out }}=\int \rho_{\text {out }} \cdot d V=0$, where $\rho_{\text {in }}$ and $\rho_{\text {out }}$ are the charge densities in and out the star.

From Eqs.(4) and (5), the charge intensity and the charges on the stellar surface is

$$
\begin{gathered}
\sigma_{\mathrm{s}}=\frac{1}{4 \pi}\left(\boldsymbol{E}_{\mathrm{out}}-\boldsymbol{E}_{\mathrm{in}}\right)_{r=r_{0}} \cdot \hat{r}=\frac{3 \Omega r_{0} B_{0} \sin ^{2} \theta}{8 \pi c}, \\
Q_{\mathrm{s}}=\int_{r=r_{0}} \sigma_{\mathrm{s}} \cdot d s=\frac{r_{0}^{3} \Omega B_{0}}{c}
\end{gathered}
$$

where $\theta$ is the polar angular between arbitrary vector $\boldsymbol{r}$ and $\hat{k}$.

From the calculation above, we can obtain the total charges

$$
\left.Q_{\text {total }}=Q_{\text {in }}+Q_{\text {out }}+Q_{\mathrm{s}}=\frac{2 \pi R_{0}^{3} B_{0}}{3 P c} \simeq 2.3 \times 10^{10} \frac{r_{6}^{3} B_{12}}{P} \text { (coulombs }\right)
$$

where $r_{6}=r_{0} /\left(10^{6} \mathrm{~cm}\right), B_{12}=B_{0} /\left(10^{12} \mathrm{G}\right)$. From this equation, it is shown that the aligned pulsars should be positively charged.

It is evident that this total charge in Eq. (9) is different from that obtained in Eq. (17) of Xu et al. (2006, $\left.Q \sim 10^{-3} r_{6}^{3} B_{12} / P^{2}\right)$. This difference could result in a shift of the boundary (i.e., the field lines being at the same electric potential as the interstellar medium) which separating regions I and II in Fig. 1 of Xu et al. (2006): the boundary should be closer (i.e., above the critical field lines) to the magnetic axis if the real charge is greater than $Q$. Unfortunately, we are faraway from obtaining an exact value of charge since a global solution to pulsar magnetosphere is still impossible. 

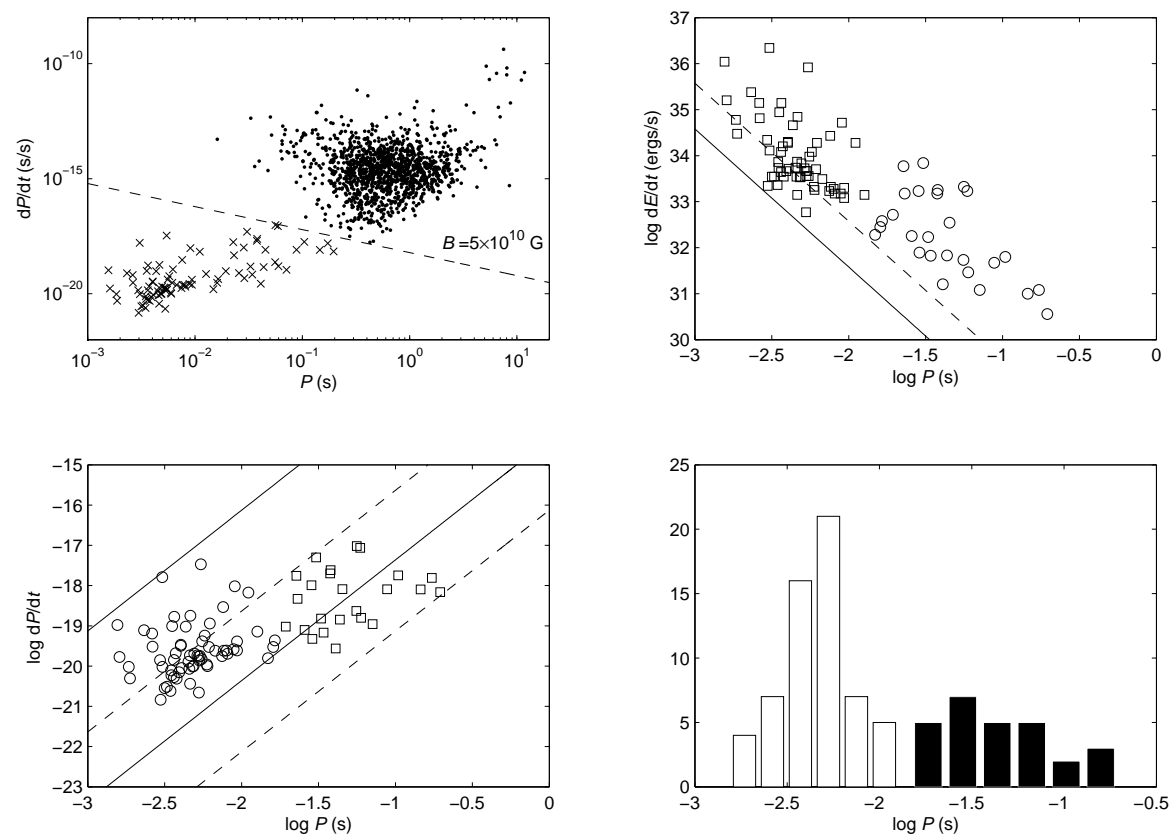

Fig. 1 Left upper panel: Period derivatives $\dot{P}$ as a function of period $P$ for 1349 radio pulsars. The dots represent the normal radio pulsars with $B>B_{0}$ and the stars are the millisecond radio pulsars with $B<B_{0}$ if the dividing line of them is the magnetic field $B_{0}=5 \times 10^{10} \mathrm{G}$. Left lower panel: The period derivative $\dot{P}$ as a function of period $P$ for millisecond radio pulsars. The empty circles (Group I') and squares (Group $I^{\prime}$ ) are two groups divided by Kmeans methods. The regions between two solid lines(for Group I') and dash lines (Group $I I^{\prime}$ ) represent limit ranges of radii for these two groups, respectively. Right upper panel: Spin down energy loss rate $\dot{E}$ as a function of period $P$ for millisecond radio pulsars. The empty circles (Group $I$ ) and squares (Group II) are two groups divided by Kmeans methods. The solid and dash lines represent the minimums of radius for these two groups. Right lower panel: Two-peak period $P$ distribution of 87 millisecond radio pulsars.

\section{EVIDENCE FOR LOW-MASS MILLISECOND PULSARS}

There are 1394 observational pulsars ${ }^{1}$ with known $P$ and $\dot{P}$ (thus $\dot{E}$ ) simultaneously. The numbers of millisecond and normal radio pulsars are 87 and 1307 if the dividing lines is by an "apparent" magnetic field of $B_{0}=6.4 \times 10^{19} \sqrt{P \dot{P}} \mathrm{G}=5 \times 10^{10} \mathrm{G}$ as shown in left upper panel of Fig.1. Here we only consider 87 millisecond pulsars $\left(B<B_{0} \mathrm{G}\right)$.

It is known that the numerical value of rotation energy loss rate

$$
\dot{E}=-I \Omega \dot{\Omega} \simeq 3.1 \times 10^{25}\left(\frac{r_{6}}{10^{6}}\right)^{2} \frac{\dot{P}_{-20}}{10^{-20}} P^{-3},
$$

\footnotetext{
${ }^{1}$ http://www.atnf.csiro.au/research/pulsar/psrcat/
} 
Table 1 The radius and mass ratios of Group I to Group II from the relation of $P-\dot{E}$ and that of Group I' to Group II' from the relation of $P-\dot{P}$.

\begin{tabular}{ccc}
\hline Relation & Radius Radio & Mass Ratio \\
\hline$P-\dot{E}$ & 0.32 & 0.03 \\
$P-\dot{P}$ & 0.01 & 0.13 \\
\hline
\end{tabular}

where rotational inertia of star $I \simeq \frac{2}{5} M r_{6}^{2}$ (the star is assumed to be a homogeneous rigid sphere), $M=M_{\odot}, \dot{P}_{-20}=\dot{P} / 10^{-20}$. Note that the pulsar radius could be a variable. A potential drop across the open field line region for a rotating magnetic pulsar could be simply

$$
\Phi=6.6 \times 10^{12} B_{12} r_{6}^{3} P^{-2} \quad \mathrm{~V} .
$$

For giving the hints about the star radius and showing possible evidence for low-mass millisecond radio pulsars in the bare strange star model by observational data, we investigate the relations of $\dot{E}-P$ and $\dot{P}-P$ as shown in left lower panel and right upper panel of Fig.1. Secondly, applying the Kmeans method, we divide them into two groups: 60 pulsars in Group I and 27 pulsars in Group II; 63 in Group I' and 24 in Group II'. Thirdly, from $B=6.4 \times 10^{19} \sqrt{P \dot{P}}$ $\mathrm{G}$ and assign $\dot{P}_{-20}=1.0, \Phi=1.0 \times 10^{12} \mathrm{~V}$ in Eqs. (10) and (11) for 87 millisecond pulsars, we give the minimums of radius for these two groups $r_{6 I} \approx 0.35$ and $r_{6 I I} \approx 1.1$ and the limit ranges of radii $0.065 \leq r_{6 I^{\prime}} \leq 0.35$ and $0.17 \leq r_{6 I I^{\prime}} \leq 0.65$. Finally, from the analysis above and according to Eq.(10) in Xu (2005), we can obtain the radius and mass ratios of Group I to Group II from the relation of $P-\dot{E}$ and those of Group I' to Group II' from the relation of $P-\dot{P}$ as shown in Table 1 .

\section{CONCLUSIONS}

The total charge of an uniformly magnetized aligned pulsar with Goldreich-Julian density outside the star is calculated. The boundary which separates the positive and negative flows in a magnetosphere is not necessary the critical lines if the real charge is not that given by Xu et al. (2006). The statistical relation between the pulsar's $\dot{E}$ (or the period derivative $\dot{P}$ ) and the period $P$ may indicate that the millisecond radio pulsars with small periods could be low-mass bare strange stars.

Acknowledgments: The authors thank helpful discussion with the members in the pulsar group of Peking University. This work is supported by NSFC (10273001, 10573002), the Special Funds for Major State Basic Research Projects of China (G2000077602), and by the Key Grant Project of Chinese Ministry of Education (305001).

\section{References}

Arons J., Scharlemenn E. T., 1979, ApJ, 231, 854

Cheng K. S., Ho C., Ruderman M., 1986, ApJ, 300, 500

Goldreich P., Julian W. H., 1969, ApJ, 157, 869

Qiao G. J., Lee K. J., Wang H. G., Xu R. X., Han J. L., 2004, ApJ, 606, L49

Ruderman M. A., Sutherland P. G., 1975, ApJ, 196, 51

Xu R. X., 2005, MNRAS, 356, 359

Xu R. X., Cui X. H., Qiao G. J., 2006, ChJAA, in press 
This paper was prepared with the ChJAA LATEX macro v1.0. 